\title{
Impact of Ocular Residual Astigmatism On Anterior Corneal Astigmatism In Children With Low And Moderate Myopia
}

Jian Lin

Lianyungang Maternal and Child Health Hospital

Dexiang An

Lianyungang Maternal and Child Health Hospital

Yun Lu

Lianyungang Maternal and Child Health Hospital

Dongmei Yan ( $\Delta$ 596751675@qq.com )

Lianyungang Maternal and Child Health Hospital

\section{Research Article}

Keywords: ocular residual astigmatism, anterior corneal astigmatism, orthokeratology, myopia.

Posted Date: December 15th, 2021

DOI: https://doi.org/10.21203/rs.3.rs-1099651/v1

License: (a) (i) This work is licensed under a Creative Commons Attribution 4.0 International License.

Read Full License 


\section{Abstract}

Background: To assess the influence of ocular residual astigmatism to anterior corneal astigmatism in children with low and moderate myopia.

Methods: Refractive astigmatism was obtained by subjective manifest refraction. Anterior corneal astigmatism was obtained by IOL Master. Using Thibos vector analysis to calculate ocular residual astigmatism. Correlation analysis was used to assess the relationship between the magnitude of ocular residual astigmatism and anterior corneal astigmatism. The influence of ocular residual astigmatism to anterior corneal astigmatism was evaluated by Physical method.

Results: The study analyzed 241 right eyes of 241 children aged 8 to 18 years old. In this study, the median magnitude of ocular residual astigmatism was $1.02 \mathrm{D}$, with interquartile range was $0.58 \mathrm{D}$. Against-the-rule ocular residual astigmatism was seen in 232 eyes (96.3\%). There was a significant and moderate correlation between ocular residual astigmatism and anterior corneal astigmatism $(r=0.50, P<$ 0.001). The ocular residual astigmatism in 240 eyes (99.6\%) had a compensatory effects on anterior corneal astigmatism. The mean compensation value was $1.00 \pm 0.41 \mathrm{D}$ (rang 0.02 D to 2.34 D). Based on this effect, 37 eyes had different axial classification of anterior corneal astigmatism and refractive astigmatism. By contrast, one eye $(0.4 \%)$ had oblique ocular residual astigmatism and superimposed with-the-rule anterior corneal astigmatism.

Conclusions: The magnitude of ocular residual astigmatism was relatively huge in myopia children and predominantly compensated anterior corneal astigmatism. The ocular residual astigmatism should be assessed first before fitting orthokeratology.

\section{Background}

Astigmatism is a common optical defect and prevails in human eyes. ${ }^{1-3}$ It is defined as the difference in power between the steep and flat ocular meridians, which causes each point from an object is refracted into two line foci with specific orientations. ${ }^{2}$ Significant astigmatism ( $\geq 1.0 \mathrm{D}$ ) reduces visual acuity, interferes with visual development and causes various symptoms like glare, monocular diplopia, asthenopia, and distortion. ${ }^{2,4}$ Children with against-the-rule (ATR) astigmatism had higher risk of myopia than those with with-the-rule (WTR) astigmatism. ${ }^{5}$ It is more difficult to treat astigmatism compared to other refractive errors.

Refractive astigmatism (RA), anterior corneal (ACA) and ocular residual astigmatism (ORA) are different types of astigmatism. RA is the result of the combination of ACA and ORA. The ORA is composed of posterior corneal surface, crystalline lens, a lesser extent vitreous, retina with the perceptual physiology. ${ }^{1,3,6}$ ORA were frequently calculated by the vectorial difference between RA and ACA. Previously studies had shown that ACA was mainly WTR astigmatism, ${ }^{3}$ whereas most of the ORA 
showed ATR astigmatism. ${ }^{3,7}$ So it is generally believed that the ORA provided a compensatory effects for ACA. ${ }^{8-10}$ But so far, there are few detailed informations on these effects.

Myopia is the most common ametropia, especially in school-aged children. Modern overnight orthokeratology can diminish refractive error rapidly, reliably, and reversibly. ${ }^{11}$ It had been widely accepted due to safe, effective, reversible. The essence of orthokeratology is to shape the corneal epithelium. To be specific, orthokeratology causes central corneal epithelium thinning and mid-peripheral cornea epithelium thickening, ${ }^{12-14}$ while without changing posterior corneal radius. ${ }^{15-16}$ Multiple studies have

demonstrated that orthokeratology can reduce the ACA significantly. ${ }^{17-19}$ As a result, the ORA was exposed after orthokeratology treatment. As mentioned previously, ORA is mainly ATR astigmatism, which has higher risk of myopia than RA (mainly WTR astigmatism). On the other hand, it's important to understand the course of the eye's refraction to make clear distribution of the astigmatism components. ${ }^{2,3}$ The purpose of this study was investigate the distributional characteristics of various types of astigmatism and assess the specific effect of ORA to ACA in children with orthokeratology indications. To provide data for improving the effectiveness of orthokeratology treatment.

\section{Materials And Methods}

This study followed the tenets of the Declaration of Helsinki and was approved by Lianyungang Maternal and Child Health Hospital review board. Informed consent was obtained from at least one parent of all participating children after explanation of the nature of the study.

\section{Participants Selection}

This cross-sectional study was conducted on 241 eyes of 241 subjects aged 8-18 years. Patients were included in this study if they had myopic from -5.00 to $-1.00 \mathrm{D}$ and regular astigmatism between $-3.00 \mathrm{D}$ to $-0.25 \mathrm{D}$ (ATR and oblique astigmatism are no less than $-2.00 \mathrm{D}$ ), and best corrected monocular visual acuity $20 / 20$ or better. The exclusion criteria included any organic diseases of eyes such as cataract, glaucoma, keratoconus, irregular astigmatism, nystagmus and children with strabismus. When RA was 0 , it is unable to determine the properties of RA's axis. Therefore, they were also excluded. Finally, a total of 241 children met the inclusion criteria: 102 females and 139 males. The mean age was $11.8 \pm 2.2$ years. Only right eyes data are taken for analysis.

\section{Examination protocol and collect parameters}

Standard subjective refraction tests were performed, and the RA was received by subjective manifest refraction. The ACA was the power difference between the steep and flat meridians on the anterior corneal surface. The IOL-Master 500 (Carl Zeiss, Meditec AG Jena, Germany) was used to measure anterior corneal curvature. Multiplying the curvature by 0.3375 to calculate corneal power. Three consistent measurements were collected and the averages were analysed. ${ }^{3}$ 


\section{Data Analysis And Calculations}

As described in our previous article ${ }^{20}$ the positive cylinder notation is more consistent with the laws of physics and mathematics. So both RA and ACA are converted into the positive-cylinder notation before calculation. In addition, RA was transformed into corneal plane before calculating ORA.

RA and ACA were transformed into power vector components using Thibos method: ${ }^{21}$

$$
\begin{aligned}
& J_{0(\Omega A)}=-\frac{R A}{2} \times \operatorname{COS}\left(2 \beta_{R A}\right), \quad J_{4 Q \Omega Q t}=-\frac{R A}{2} \times \operatorname{SIN}\left(2 \beta_{R A}\right) \\
& J_{0(A C A)}=-\frac{A C A}{2} \times \operatorname{COS}\left(2 \beta_{A C A}\right), \quad J_{45(A C A)}=-\frac{A C A}{2} \times \operatorname{SIN}\left(2 \beta_{A C A}\right)
\end{aligned}
$$

where $\mathrm{J}_{0}$ and $\mathrm{J}_{45}$ were the horizontal/vertical and oblique components of astigmatism, respectively, and $\beta$ was the positive-cylinder axis.

The components of ORA were determined as:

$$
J_{O(O R A)}=J_{O(R A)}-J_{O(A C A)}, \quad J_{45(O R A)}=J_{45(R A)}-J_{45(A C A)}
$$

So the magnitude and axis ( $\left.\beta_{\text {ORA }}\right)$ of ORA were calculated as:

$$
O R A=2 \sqrt{J_{\text {QORA })}^{2}+J_{4 \& O R A)}^{2}}, \quad 2 \beta_{\text {ORA }}=\arctan \left(\frac{J_{4 S(O R A)}}{J_{\text {O(ORA) }}}\right)
$$

According to the vector relationship of ORA, ACA and RA at the double angle vector diagram, the only corresponding positive cylinder axis of ORA $\left(\beta_{\text {ORA }}\right)$ was defined. ${ }^{20}$

For describing the distribution of astigmatic axes, with-the-rule astigmatism was determined as positivecylinder axes from $60^{\circ}$ to $120^{\circ}$, and against-the-rule astigmatism as positive-cylinder axes from $1^{\circ}$ to $30^{\circ}$ or $150^{\circ}$ to $180^{\circ}$. Oblique astigmatism was defined as positive-cylinder axes from $31^{\circ}$ to $59^{\circ}$ or $121^{\circ}$ to $149^{\circ}$.

\section{Analysis Process Of The Effect Of Ora To Aca}

When the difference of vector angle between ORA and ACA was greater than $90^{\circ}$ on the double angle vector diagram, ORA will compensate ACA. ${ }^{20}$ The compensation values (CV) were calculated by multiplying ORA by cosine $\left(180^{\circ}-\mathrm{a}\right)$, where $\mathrm{a}\left(\right.$ rang $90^{\circ}$ to $\left.180^{\circ}\right)$ was the included angle between vector of ORA and ACA on the double angle vector diagram (Figure 1).

\section{Statistical methods}


SPSS statistics software package version 17.0 for Windows (IBM, Armonk, NY, USA) was used for the statistical analysis and calculations. Normality of all data samples was checked by means of the Kolmogorov-Smirnov test. The magnitude of RA, ORA, spherical refraction and spherical equivalent refraction (SER) were non-normally distributed. The non-normality measurement data were expressed as median value and interquartile range (IQR). The magnitude of ACA was normally distributed and was expressed as mean \pm standard deviation (SD). Correlation coefficients (Pearson or Spearman depending if normality condition could be assumed) were used to assess the correlation between ORA and ACA, ORA and RA. Correlations were considered to be statistically significant when the associated $p$-value was $₫ 0.05$.

\section{Results}

\section{Characteristics of the study population}

Of 241 patients (241 right eyes), 139 (57.7\%) were male. Mean age of patients was 11.8 years old (SD: 2.2; range: 8 - 18 years). The sphere refraction ranged $-5.00 D$ to $-1.00 D$ (median value was $-3.00 D$, IQR was $2.0 \mathrm{D}$ ). The myopia was -3.00 or less in $54.8 \%$ (132 eyes). The SER ranged $-6.25 \mathrm{D}$ to $-1.13 \mathrm{D}$ (median value was $-3.38 \mathrm{D}$, IQR was $2.0 \mathrm{D}$ ). At the corneal plane, the ACA was 1.00 or more in $85.5 \%$ (206 eyes) with mean ACA was $1.63 \pm 0.62$ (range $0.25 \mathrm{D}$ to $3.54 \mathrm{D}$ ). The RA ranged $0.22 \mathrm{D}$ to $2.63 \mathrm{D}$ (median value was $0.49 \mathrm{D}$, IQR was $0.46 \mathrm{D}), 48$ eyes $(19.9 \%)$ were $1.00 \mathrm{D}$ or more RA. The ORA ranged $0.28 \mathrm{D}$ to $2.48 \mathrm{D}$ (median value was 1.02 D, IQR was $0.58 \mathrm{D}$ ) and it was 1.00 or more in $51.5 \%$ (124 eyes). Table 1 indicates the patient's characteristics.

Table 1. Patient's characteristics

\begin{tabular}{|lll|}
\hline Variable & Mean \pm SD/Median(IQR) & Rang \\
\hline Age (y) & $11.8 \pm 2.2$ & $8-18$ \\
\hline Spherical refraction (D) & $-3.00(2.00)$ & $-5.00--1.00$ \\
RA (D) & $0.49(0.46)$ & $0.22-2.63$ \\
ACA (D) & $1.63 \pm 0.62$ & $0.25-3.54$ \\
ORA (D) & $1.02(0.58)$ & $0.28-2.48$ \\
SER (D) & $-3.38(2.00)$ & $-6.25--1.13$ \\
\hline
\end{tabular}

$\mathrm{RA}=$ refractive astigmatism; $\mathrm{ACA}=$ anterior corneal astigmatism;

ORA = ocular residual astigmatism; SER= spherical equivalent refraction

\section{Distribution of astigmatism}

Figure 2 shows the distributions of astigmatism. The prevalence of RA ( $\geq 1.0 \mathrm{D})$ was $19.9 \%, A C A(\geq 1.0$ D) $85.5 \%$, and ORA ( $\geq 1.0$ D) $51.5 \%$. ACA had peak prevalence $(61.8 \%$ ) between $1.0 \mathrm{D}$ to $2.0 \mathrm{D}$ (including 
1D, excluding 2D). RA displayed peak prevalence (80.1\%) less than 1.0 D. ORA shown two peak prevalence $(48.5 \%$ less than $1.0 \mathrm{D}$ and $49.8 \%$ between $1.0 \mathrm{D}$ to $2.0 \mathrm{D})$. With respect to axes, WTR ACA was observed in 235 eyes (97.6\%), ATR ACA was seen in 2 eyes (0.8\%), and oblique ACA was discovered in 4 eyes (1.6\%). 202 (83.8\%) eyes shown WTR RA, 21 (8.7\%) shown ATR RA, and 18 (7.5\%) eyes shown oblique RA. 232 eyes (96.3\%) had ATR ORA, 2 eyes $(0.8 \%)$ were WTR ORA, 7 eyes $(2.9 \%)$ shown oblique ORA.

\section{The relationship of ORA and ACA, ORA and RA.}

There was no significant correlations between the magnitude of ORA and RA ( $r=-0.05, P=0.42)$. There was a significant and moderate correlations between the magnitude of ORA and ACA $(r=0.50, P<0.001)$. The predicting equations of the ORA from the magnitude of ACA were obtained (Figure 3 ):

ORA $=0.54+0.31 \times A C A\left(R^{2}=0.23\right)$

\section{The effects of ORA to ACA}

The effects of ORA on ACA was analyzed and found the ORA of 240 eyes (99.6\%) had a compensatory effects on ACA. Of them, 233 eyes (97.1\%) shown ATR ORA, 5 eyes (2.1\%) had oblique ORA, and 2 eyes $(0.8 \%)$ were WTR ORA. The mean compensation values (CV) was $1.00 \mathrm{D}$ (SD:0.41 D, rang $0.02 \mathrm{D}$ to 2.34 D). The magnitude of CV/ACA was 0.25 or less in 14 eyes, 0.50 or less in 63 eyes, 0.75 or less in 179 eyes, and exceeded 1.00 in 16 eyes (Table 3). By contrast, only one eye shown superimposed effect on ACA and had oblique ORA (ORA was $0.67 \times 139$ ). The superimposition value was $0.15 \mathrm{D}$, with ACA was $0.75 \times 101$. After the compensation effects of ORA to ACA, the axial classification of ACA and RA were different in 37 eyes. Specifically, shift in axis from WTR ACA to ATR RA in 17 eyes and to oblique RA in other 17 eyes, shift in axis from oblique ACA to WTR RA in 1 eye and to with ATR RA in 2 eyes. 204 eyes were the same axial classification of ACA and RA. To be specific, 201 eyes were WTR ACA and RA, 2 eyes had ATR ACA and RA, and 1 eye shown oblique ACA and RA.

\section{The effects of ORA to WTR ACA}

In this study, WTR ACA was seen in 235 eyes. Of them, 230 eyes (97.9\%) had ATR ORA, 5 eyes $(2.1 \%)$ were oblique ORA. The ORA in 234 eyes (99.6\%) had a compensatory effects on WTR ACA. Of them, 230 eyes (98.3\%) shown ATR ORA, 4 eyes (1.7\%) were oblique ORA. The mean compensation values was 1.01 $\mathrm{D}(\mathrm{SD}: 0.40 \mathrm{D}$, rang $0.20 \mathrm{D}$ to $2.34 \mathrm{D}$ ). The magnitude of CV/ACA was 0.25 or less in 13 eyes, 0.50 or less in 61 eyes, 0.75 or less in 174 eyes, and exceeded 1.00 in 15 eyes (Table 3 ). In the other hand, one eye with oblique ORA shown superimposed effect on WTR ACA.

\section{The effects of ORA to ATR and oblique ACA}

One eye with oblique ORA and one eye with WTR ORA played a compensatory effects on ATR ACA. Three eyes with ATR ORA and one eye with WTR ORA exhibited compensatory effects on oblique ACA (Table 2). 
Table 2. The effects of ORA to ACA ( $\mathrm{N}=241)$.

\begin{tabular}{|c|c|c|c|c|c|c|}
\hline & \multicolumn{6}{|l|}{ ORA } \\
\hline & \multicolumn{3}{|c|}{ Compensatory effects } & \multicolumn{3}{|c|}{ Superimposed effects } \\
\hline & WTR & ATR & Oblique & WTR & ATR & Oblique \\
\hline WTR ACA $(n=235)$ & 0 & 230 & 4 & 0 & 0 & 1 \\
\hline ATR ACA $(n=2)$ & 1 & 0 & 1 & 0 & 0 & 0 \\
\hline Oblique ACA $(n=4)$ & 1 & 3 & 0 & 0 & 0 & 0 \\
\hline
\end{tabular}

Table 3. Distributions of the ratio of CV/ACA $(\mathrm{N}=240)$.

\begin{tabular}{|llllll|}
\hline \multicolumn{7}{|l|}{ The ratio of CV/ACA } & & \\
& $\leq 0.25(n)$ & $\leq 0.50(n)$ & $\leq 0.75(n)$ & $\leq 1.00(n)$ & $>1.00(n)$ \\
\hline WTR ACA $(n=234)$ & 13 & 61 & 174 & 219 & 15 \\
\hline ATR ACA $(n=2)$ & 0 & 0 & 2 & 2 & 0 \\
\hline Oblique ACA $(n=4)$ & 1 & 2 & 3 & 3 & 1 \\
\hline
\end{tabular}

$\mathrm{CV}$ :compensation values

\section{Discussion}

In the subjects of low and moderate myopia, the prevalence of RA ( $\geq 1.0 \mathrm{D})$ was $19.9 \%, A C A(\geq 1.0 \mathrm{D})$ $85.5 \%$, and ORA ( $\geq 1.0 \mathrm{D}$ ) $51.5 \%$. The mean ACA was $1.62 \mathrm{D}$ (SD $0.62 \mathrm{D}$ ), the median(IQR) RA was 0.49 (0.46), and the median(IQR) ORA was 1.02 (0.58). Compared with the whole categories of refractive states (including emmetropia and ametropia), the prevalence of RA $\geq 1.0 \mathrm{D}(19.9 \%)$ was similar to those reported in central China $(17.4 \%)^{3}$ and Singapore $(19.2 \%)^{22}$ but lower than Hong Kong $(28.4 \%)^{23}$ and Taiwan (32.6\%). ${ }^{24}$ Whereas, the prevalence of ACA (85.5\%) and ORA (51.5\%) were significantly higher than those studies. ${ }^{3,25}$ Meanwhile, the magnitude of ORA was larger than other studies. Li et al. ${ }^{3}$ analysed 1783 12-year-old students and reported the mean ORA was $0.72 \mathrm{D}$. Huynh et al. ${ }^{8}$ found the mean ORA was $0.76 \mathrm{D}$ in 6-year-old children. One possibility reason for this result is that the magnitude of ACA and ORA of myopia was significantly larger than that of emmetropia and hyperopia. Another possibility is the compensation effects of ORA to ACA in myopia were more significantly than other refractive states.

The prevailing wisdom was that the ORA compensated ACA. ${ }^{8-10}$ Although our recent work evaluated the contribution of ORA to ACA in 5 -year-old children, ${ }^{20}$ but there are still no detailed information on the 
compensation effects in myope children. There was a significant and moderate correlations between the magnitude of ORA and ACA $(r=0.50, P<0.001)$. The ORA in 240 eyes $(99.6 \%)$ had a compensatory effects on ACA. The compensation values of $6.7 \%(16 / 240)$ eyes exceeded that of ACA. The distribution of astigmatic axes of $15.4 \%$ (37 / 240) eyes changed after the compensation effects (ie. the axial classification of ACA and RA were different). For 235 eyes with WTR ACA, 99.6\% (234 / 235) ORA worked to offset it. Both ATR and oblique ORA can counteract WTR ACA, while oblique ORA also can superimposed it. The results was similar to our recent study in in 5-year-old children with significant astigmatism. ${ }^{20}$ With regard to ATR ACA, both WTR and oblique ORA had a compensatory effects on it. WTR and ATR ORA can counteract oblique ACA.

Actually, the correlation between ORA and ACA, ORA and RA would vary according the population being studied (such as age, sphere, cylinder, etc.). We found a moderate and positive correlations between ORA and ACA $(r=0.50, P<0.001)$ in low and moderate myopic eyes. Wallerstein and colleagues ${ }^{7}$ obtained the similar outcomes $(r=0.44)$ by studying 21,580 myopic eyes. Nevertheless, our previous study found a much weaker correlation between ORA and ACA ( $r=0.17)$ by studying 14 emmetropic eyes, 68 myopic eyes, and 19 hyperopic eyes. ${ }^{20}$ Moreover, there was no significant correlations between ORA and RA ( $r=-$ $0.05, P=0.42$ ) in this study. Piñero et al. also reported a negative but no statistical significance correlation ( $r=-0.01, P=0.89$ ) by studying 14 emmetropic eyes, 68 myopic eyes, and 19 hyperopic eyes. ${ }^{6} \mathrm{~A}$ positive correlation was obtained in other studies. ${ }^{1,7}$ Our previous study found a significant and negative correlation between ORA and RA $(r=-0.27, P=0.001) .{ }^{20}$ Characteristics of the studied population, such as age, sphere, cylinder, etc, may be the reason for the different research results.

Multiple studies have demonstrated that orthokeratology causes significant changes in corneal astigmatism. ${ }^{17-19}$ Mountford and Pesudovs ${ }^{17}$ stated $87.0 \%$ of patients with reverse geometry orthokeratology lenses had some reduction of ACA in their study. Chan ${ }^{18}$ reported reductions in ACA of up to $-2.50 \mathrm{D}$ three weeks after toric orthokeratology treatment(1.5D). Chen et al. ${ }^{19}$ investigated 35 myopic children with moderate-to-high astigmatism, and found a 79 percent reduction in ACA after one month of toric orthokeratology. As mentioned previous, orthokeratology did not change the posterior cornea radius. ${ }^{15-16}$ Consequently, the ORA were exposed after orthokeratology treatment. Relatively large amounts of ORA, which is mainly against-the-rule astigmatism, existed in 12-year-old children with orthokeratology indications, it may one of the reasons to degrade visual quality after orthokeratology. As Sorbara et al. ${ }^{26}$ found that the proportion of subjects with spectacles reaching $6 / 6$ or better visual acuity was higher than those wearing orthokeratology lens ( $89 \%$ vs $83 \%$ ). Unfortunately, there is no study on the relationship between ORA and orthokeratology up to now. Future research needs to be done in the correlation between ORA and residual astigmatism after shaping with orthokeratology, and in the specific influence of ORA to visual quality after orthokeratology.

In conclusion, for low and moderate myopia eyes, we found the prevalence of ORA ( $\geq 1.0 \mathrm{D})$ was relatively high and the magnitude was huge, nearly all (99.6\%) ORA compensate ACA, the magnitude of CV/ACA exceeded 1.00 in 6.7\%(16/240) eyes, and 15.4\% (37/240) eyes had different axial classification 
of ACA and RA after the compensation effects. The ORA were exposed after orthokeratology treatment. So, the ocular residual astigmatism should be assessed first before the completion of a course of orthokeratology. In addition, more attention should be paid to the specific influence of ORA to the effective of orthokeratology.

\section{List Of Abbreviations}

ORA: ocular residual astigmatism; ACA: anterior corneal astigmatism;

RA: refractive astigmatism; WTR: with-the-rule; ATR: against-the-rule;

SER: spherical equivalent refraction; IQR: interquartile range;

SD: standard deviation; CV: compensation values;

\section{Declarations}

\section{Ethics approval and consent to participate}

Institutional Review Board (IRB)/Ethics Committee approval was obtained by Human Medical Ethics Committee of Lianyungang Maternal and Child Health Hospital. The authors confirm that the research followed the tenets of the Declaration of Helsinki and that informed consent was obtained from the subjects after explanation of the nature and possible consequences of the study.

\section{Consent for publication}

Not applicable

\section{Availability of data and materials}

The datasets used and/or analyzed during the current study are available from the corresponding author on reasonable request.

\section{Competing interests}

The authors declare that they have no competing interests

\section{Funding}

None.

\section{Authors' contributions}

$\mathrm{JL}$ was a major contributor in writing the manuscript. Acquisition, analysis or interpretation of data: JL, DM Y. Data collection, editing: YL, DX A. Technical or material support: YL, DX A. Study 
supervision: DM Y. All authors read and approved the final manuscript.

\section{Acknowledgments}

Not applicable

\section{References}

1.Mohammadpour M, Heidari Z, Khabazkhoob M, Amouzegar A, Hashemi H. Correlation of major components of ocular astigmatism in myopic patients. Contact Lens and Anterior Eye. 2016; 39(1): 2025.

2.Kee CS. Astigmatism and its role in emmetropization. Exp Eye Res. 2013;114:89-95.

3.Li H, Li SM, Liu LR, et al. Astigmatism and its components in 12-year-old Chinese children: the Anyang Childhood Eye Study. Br J Ophthalmol. 2018; 0:1-7.

4.Thornton SP. Cataracts and the surgical control of astigmatism. J Cataract Refract Surg. 1989;15,11.

5.Gwiazda J, Grice K, Held R, McLellan , Thorn F. Astigmatism and the development of myopia in children. Vision Res. 2000;40:1019-1026.

6.Piñero DP, Ruiz-Fortes P, Pérez-Cambrodí RJ, Mateo V, Artola A. Ocular residual astigmatism and topographic disparity vector indexes in normal healthy eyes. Contact Lens \& Anterior Eye.2014; 37(1): 4954 .

7.Wallerstein, A., Gauvin, M., Qi, S. R. \& Cohen, M. Effect of the Vectorial Difference Between

Manifest Refractive Astigmatism and Anterior Corneal Astigmatism on Topography-Guided LASIK Outcomes. J. Refract. Surg. 2020;36:449-458.

8.Huynh SC, Kifley A, A.Rose K, Morgan I, Z. Heller G, Mitchell P. Astigmatism and its components in 6year-old children, Invest Ophthalmol Vis Sci. 2006; 47: 55-64.

9.Muftuoglu O, Erdem U. Evaluation of internal refraction with the optical path difference scan, Ophthalmology. 2008; 115: 57-66.

10.Ho JD, Liou SW, Tsai RJ, Tsai CY. Effects of aging on anterior and posterior corneal astigmatism. Cornea. 2010;29:632-637.

11.Nti AN. Berntsen DA. Optical changes and visual performance with orthokeratology. Clin Exp Optom. 2020; 103: 44-54.

12.Zhong X, Chen X, Xie RZ et al. Differences between overnight and long-term wear of orthokeratology contact lenses in corneal contour, thickness, and cell density. Cornea. 2009; 28: 271-279. 
13. Alharbi A, Swarbrick HA. The effects of overnight orthokeratology lens wear on corneal thickness. Invest Ophthalmol Vis Sci. 2003; 44: 2518-2523.

14. Wang J, Fonn D, Simpson TL, et al. Topographical thickness of the epithelium and total cornea after overnight wear of reverse-geometry rigid contact lenses for myopia reduction. Invest Ophthalmol Vis Sci. 2003; 44: 4742-4746.

15.Yoon JH, Swarbrick HA. Posterior corneal shape changes in myopic overnight orthokeratology. Optom Vis Sci. 2013; 90: 196-204.

16. Chen D, Lam AK, Cho P. Posterior corneal curvature change and recovery after 6 months of overnight orthokeratology treatment. Ophthalmic Physiol Opt. 2010; 30: 274-280.

17.Mountford J, Pesudovs K. An analysis of the astigmatic changes induced by accelerated orthokeratology. Clin Exp Optom. 2002; 85: 284-293.

18. Chan B, Cho P, de Vecht A. Toric orthokeratology: a case report. Clin Exp Optom 2009; 92: 387-391.

19. Chen C, Cheung SW, Cho P. Myopia control using toric orthokeratology (TO-SEE study). Invest Ophthalmol Vis Sci. 2013; 54: 6510-6517.

20.Lin J.The contribution of ocular residual astigmatism to anterior corneal astigmatism in refractive astigmatism eyes. Scientific Reports. 2021;11:10-18.

21.Thibos LN, Wheeler W, Horner D. Power vectors: An application of Fouriere Analysis to the description and statistical analysis of refractive error. Optom Vis Sci.1997;74(6):367-375.

22. Tong L, Saw SM, Carkeet A, et al. Prevalence rates and epidemiological risk factors for astigmatism in Singapore school children. Optom Vis Sci. 2002;79:606-13.

23.Leung TW, Lam AK, Deng L, et al. Characteristics of astigmatism as a function of age in a Hong Kong clinical population. Optom Vis Sci. 2012;89:984-92.

24.Shih YF, Hsiao CK, Tung YL, et al. The prevalence of astigmatism in Taiwan schoolchildren. Optom Vis Sci. 2004;81:94-8.

25.Huynh SC, Kifley A, Rose KA, et al. Astigmatism in 12-year-old Australian children: comparisons with a 6-year-old population. Invest Ophthalmol Vis Sci. 2007;48:73-82.

26. Sorbara L, Fonn D, Simpson T et al. Reduction of myopia from corneal refractive therapy. Optom Vis Sci. 2005; 82: 512-518. 
Figures
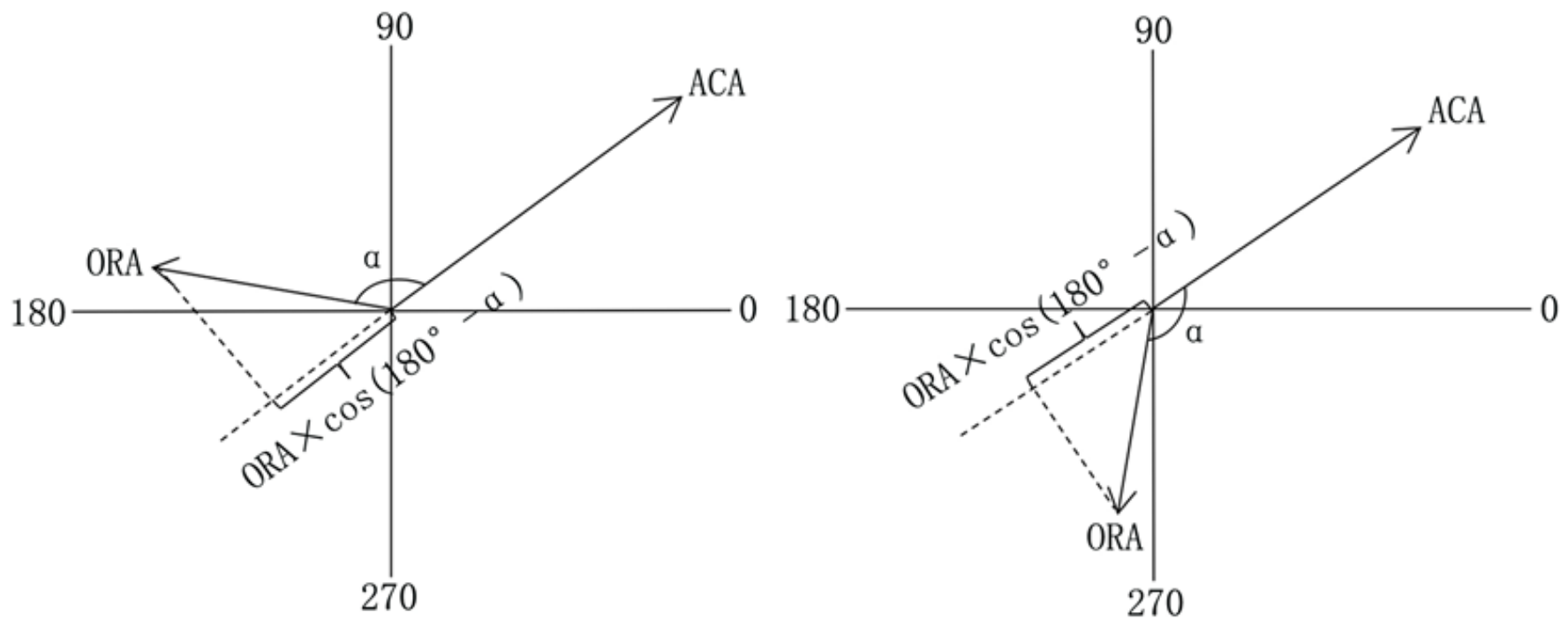

\section{Figure 1}

Calculation process of the compensation values.
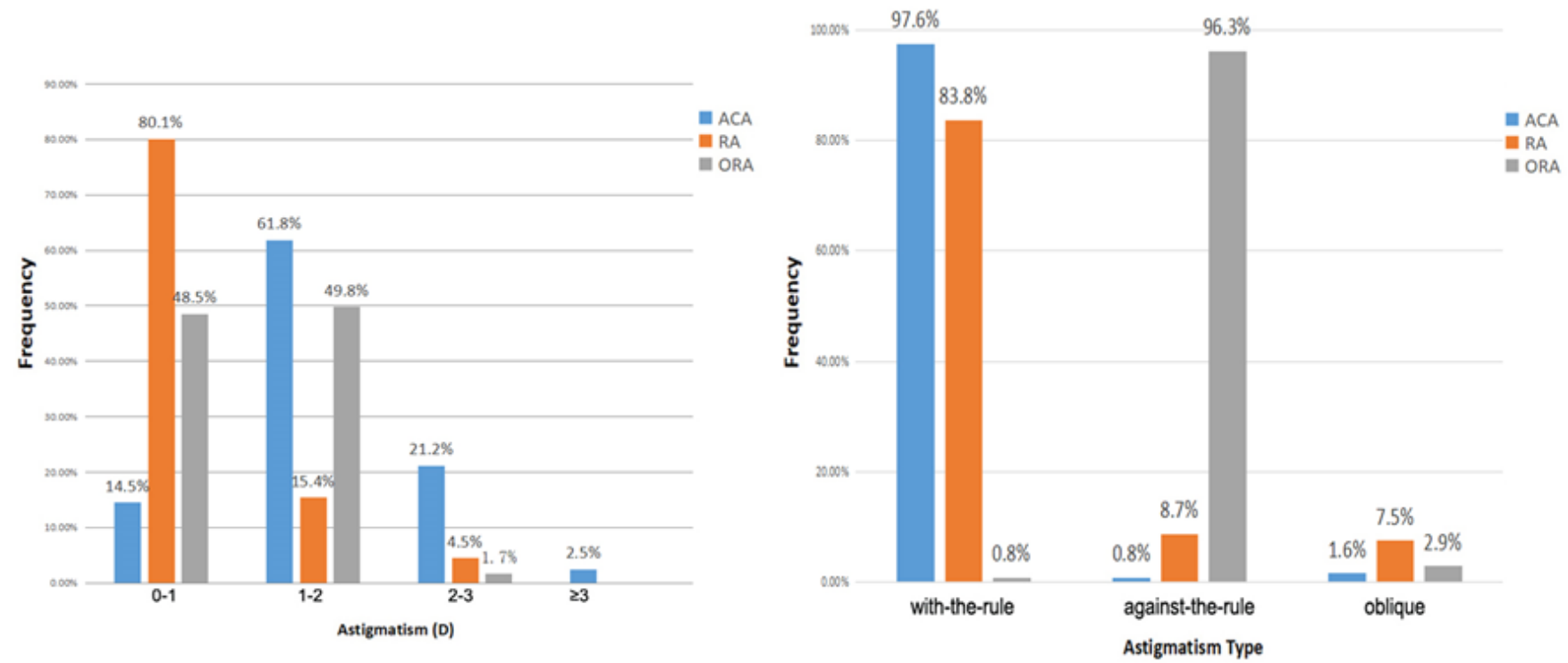

Figure 2

Distributions of different types of astigmatism. 


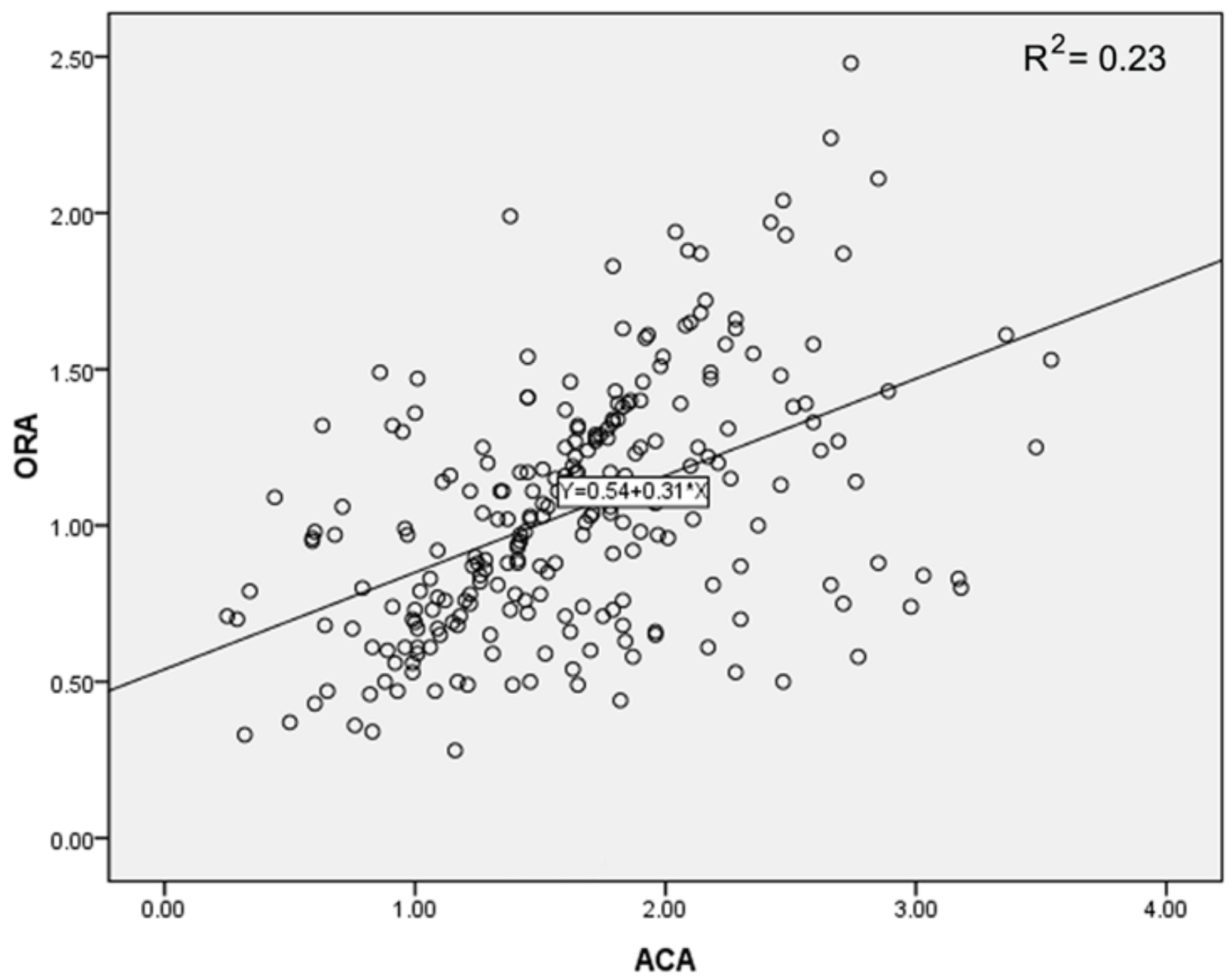

Figure 3

Correlation between the magnitude of ORA and ACA. 